\title{
Effect of gap junctions on the firing patterns and synchrony for different external inputs in the striatal fast-spiking neuron network
}

\author{
Mingming Zhang ${ }^{\mathrm{a}}$, Zongya Zhao ${ }^{\mathrm{a}}$, Ping He ${ }^{\mathrm{b}}$ and Jue Wang ${ }^{\mathrm{a},{ }^{*}}$ \\ ${ }^{a}$ The Key Laboratory of Biomedical Information Engineering of Ministry of Education, and Institute of \\ Biomedical Engineering, School of Life Science and Technology, Xi'an Jiaotong University; National \\ Engineering Research Center of Health Care and Medical Devices, Xi'an Jiaotong University Branch, \\ Xi'an 710049, P.R. China \\ ${ }^{b}$ Department of Biomedical, Industrial and Human Factors Engineering, Wright State University, \\ Dayton, Ohio, USA
}

\begin{abstract}
Gap junctions are the mechanism for striatal fast-spiking interneurons (FSIs) to interconnect with each other and play an important role in determining the physiological functioning of the FSIs. To investigate the effect of gap junctions on the firing activities and synchronization of the network for different external inputs, a simple network with least connections and a Newman-Watts small-world network were constructed. Our research shows that both properties of neural networks are related to the conductance of the gap junctions, as well as the frequency and correlation of the external inputs. The effect of gap junctions on the synchronization of network is different for inputs with different frequencies and correlations. The addition of gap junctions can promote the network synchrony in some conditions but suppress it in others, and they can inhibit the firing activities in most cases. Both the firing rate and synchronization of the network increase along with the increase of the electrical coupling strength for inputs with low frequency and high correlation. Thus, the network of coupled FSIs can act as a detector for synchronous synaptic input from cortex and thalamus.
\end{abstract}

Keywords: Network, fast-spiking interneurons, synchronization, firing rate, small-world

\section{Introduction}

Striatum is the major input nucleus of base ganglia and plays an important role in controlling the input from cortex and thalamus [1]. The fast-spiking interneurons (FSIs), as the major type of striatal interneurons, can delay or inhibit the generation of action potentials of the medium spiny neurons (MSNs), the only output neurons of striatum, resulting in regulating the firing patterns of MSNs [2-4]. The unique connections between FSIs are gap junctions by which FSIs form a sparsely connecting network [2,3] with 30 40\% probability for each FSI coupling with neighboring neurons [2,5]. The gap junctions behave as low-pass filters and the transmission efficacy of the membrane potential through

*Corresponding author: Jue Wang, School of Life Science and Technology, Xi'an Jiaotong University, Xi'an 710049, P.R. China. Tel.: +86 15332479788; Fax: +86 029 82663497; E-mail: juewang@mail.xjtu.edu.cn.

0959-2989/14/\$27.50 @ 2014 - IOS Press and the authors. 
gap junctions is frequency-dependent [6-8]. Previous physiological experiments found that FSIs represent nearly continuous firing activity and any two neurons show uncorrelated activity in awaken rodents. However, FSIs show robust task-related activity during behavioral tasks, and the temporal patterns of firing rate change are highly idiosyncratic with behavior-linked modulation of spike timing and remarkable decrease of inter-spike intervals (ISIs) $[9,10]$. These results suggest that the factors affecting the firing pattern of FSIs are especially complicated.

FSIs receive excitatory synaptic inputs from the cortex and thalamus [11] and multiple synaptic contacts on one FSI come from individual afferent fibers [12] with different conduction delay. Thus the event-related external stimuli can excite a large number of neurons in relevant brain areas which form low frequency and highly relevant inputs to FSIs. But the external disturbance only can randomly excite a small number of cortical neurons which produce high frequency and nearly uncorrelated inputs. Some studies showed that the small high-frequency signals or Gaussian white noise superposed on low-frequency signals can enhance synchronization of neuronal activities [13-15]. Hjorth reported that gap junctions can decrease the number of spikes and the high correlation among external inputs can reduce the impact of gap junctions [3].

In order to study the effect of the frequency and correlation of external inputs on the firing patterns of the neuronal network, the Gaussian white noise (WN), colored noise with more high-frequency components (HF) and colored noise with less high-frequency components (LF) were selected as external inputs. The colored noise is generated by a linear damping equation driven by white noise [16]. This paper is organized as follows: the mathematical models for FSIs and gap junctions, and the analytical approaches are introduced in Section 2; the simulation results are presented in Section 3; finally, the conclusion is shown in Section 4.

\section{Methods}

\subsection{Mathematical models}

The Izhikevich model was used as neuronal model which is a two-dimensional system of ordinary differential equation $[17,18]$ :

$$
\begin{aligned}
& \dot{v}=0.04 v^{2}+5 v+140-u+I_{e x t}+I_{g a p} \\
& \dot{u}=a(b v-u)
\end{aligned}
$$

with the auxiliary after-spike resetting:

$$
\text { If } v \geq v_{p} \text {, then } v \leftarrow c, u \leftarrow u+d
$$

where $v$ and $u$ represent the membrane potential and recovery variables respectively, $v_{p}$ is the resetting threshold, $I_{\text {ext }}$ is external input and $I_{g a p}$ is the total currents through gap junctions to each 
neuron, and parameters $a, b, c$ and $d$ define the type of neuron modeled. For FSIs, $a=0.1$, $b=0.2, c=-65$ and $d=2$.

The gap junction is modeled as a nonlinear equation proposed by Humphries which can be expressed as [19]:

$$
\begin{gathered}
\tau \dot{v}_{i j}=\left(v_{i}-v_{i j}\right)+\left(v_{j}-v_{i j}\right) \\
I_{g a p}(i)=\sum_{j=1, j \neq i}^{n} g\left(v_{i j}-v_{i}\right)
\end{gathered}
$$

where $v_{i}$ and $v_{j}$ represent the membrane potentials of the FSI pair, $v_{i j}$ is a variable associated with the gap junction, $\tau$ is the time constant for voltage decay, $I_{\text {gap }}(i)$ is the total currents injected into neuron $i, g$ is the gap junction conductance and $n$ is the total number of neurons coupling with the neuron $i$.

Different frequency sinusoidal currents were injected into FSI pair to determine suitable values for $\tau$ and $g$. When $\tau=11 \mathrm{~ms}$ and $g \leq 0.4 n S$, the coupling coefficient and phase-lag are consistent with the experimental data $[2,8,19]$. The coupling ratio is directly proportional to the conductance of gap junction whereas decreases with the increase of input frequency. But the phase-lag increases with the increase of input frequency (Figure 1(a)). The coupled neuron by the gap junction has a smaller transient response to each spike but relatively large direct current response to the sub-threshold membrane potential (see Figure 1(b)). Figure 1(c) shows the membrane potential traces of FSIs coupled with $(g \neq 0)$ (blue) and without $(g=0)$ (red) gap junctions for the same inputs. The addition of gap junctions makes the firing activities of coupled FSIs significantly different. At some time a spike is removed $(\times)$ and at other time a spike is triggered $(+)$ due to the shunting current induced by the gap junctions. These results are similar to those reported in [8].

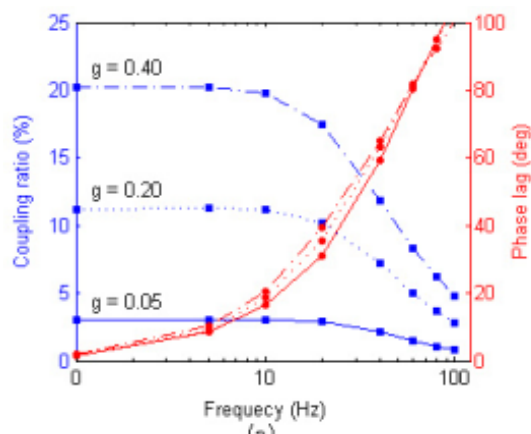

(a)

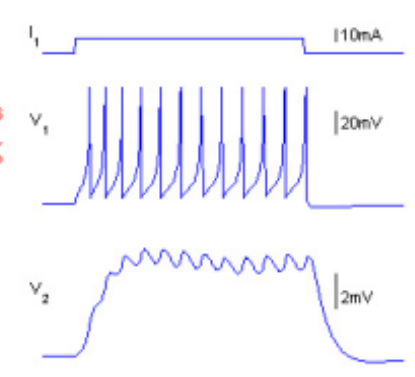

(b)

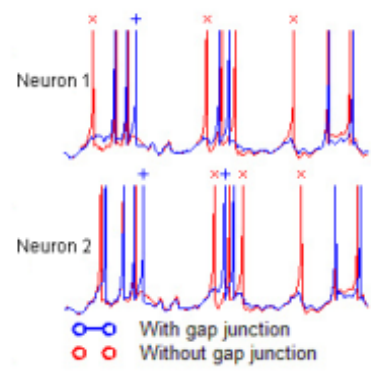

(c)

Fig. 1. The characteristics of neuronal model for FSI pair coupled with a gap junction. (a) The coupling ratio ( $\mathbf{(})$ and phase lags (•) for different frequency inputs. (b) Transmission of membrane potentials between two coupled FSIs. $I_{1}$ is the input, $V_{1}$ and $V_{2}$ are membrane potentials of the excited and coupled neurons respectively. (c) The comparison between the membrane potential traces of FSIs with (blue) and without (red) gap junctions. Top: the membrane potential traces of Neuron 1 for the same input in two cases. Bottom: the ones of Neuron 2. Two inputs for different neurons are uncorrelated. 


\subsection{Network connection}

A simple network and a small-world network were built to study the effect of gap junctions. The simple network consists of a (central) FSI coupled with multiple (peripheral) FSIs through gap junctions among which there are no direct connections (Figure 2(a)). The number of the peripheral coupled FSIs varies from 0 to 12 . The trend of firing activities and synchronization is similar but becomes obvious with the increase of the number of the coupled neurons. The typical number of the coupled neurons is 3 in the paper. The effects of gap junctions are easy to analyze in the simple network, but the complex network with more interactions can obtain results closer to those observed in experiments in vivo. The complex network is a small-word with Newman-Watts (NW) strategy (Figure 2(b)). The network is constructed by a ring of $n$ nodes, and connected to its $k$ nearest neighbors. In addition, several shortcuts are added between randomly selected pairs of nodes with a shortcut probability $p \quad(0 \leq p \leq 1)$ [20]. For simplicity, the weights of all connections are kept constant. Here we set $n=40, k=12, p=0.1$. The value of $p$ is referred to [21-23]. The FSI and the directly coupled neighboring neurons form the local network which includes 12 16 neurons for each FSI, corresponding to the $30 \sim 40 \%$ coupling probability of each FSI with others reported in $[2,5]$.

\subsection{Analysis}

The FSI spikes affected by gap junctions can be classified into three cases: spikes removed due to shunting, spikes triggered by coupled neurons and spikes appearing spontaneously [3]. The spiking change of FSIs with gap junctions $(g \neq 0)$ and those without gap junctions $(g=0)$ for the same inputs is compared. Within a period of a spike timing $\pm 2.5 \mathrm{~ms}$ in the network without gap junctions, if an spike appears in the corresponding network with gap junctions, two spikes are defined as spontaneous spikes excited by external inputs; the other spikes in the network without gap junctions are defined as removed spikes, and the other spikes in the network with gap junctions are defined as triggered spikes.

To characterize the synchronization, the synchrony measure $S y n$ is introduced [22] which can be expressed as Syn $=\Delta_{v} / \Delta$, where $\Delta_{v}=\left\langle V_{N}(t)^{2}\right\rangle_{t}-\left\langle V_{N}(t)\right\rangle_{t}^{2}, \Delta=\sum_{i=1}^{N}\left(\left\langle V_{i}(\mathrm{t})^{2}\right\rangle_{t}-\left\langle V_{i}(t)\right\rangle_{t}^{2}\right) / N$, where $V_{N}(t)=\sum_{i=1}^{N} V_{i}(t) / N$ and $\langle\cdot\rangle_{t}$ represents the average over time. The larger the value of $S y n$, the better synchronized in the network. The synchronization among the FSIs which are connected to the same neuron is defined as the local synchronization and the average of all local synchronization is defined as local Syn. The Syn of all neurons in the entire network is called global Syn .

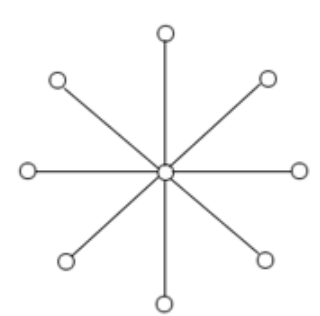

(a)



(b)

Fig. 2. Neuronal networks of FSIs. (a) The simple network. (b) The NW small-world network. 


\section{Results}

\subsection{The frequency of external inputs can influence the effect of gap junctions}

In order to analyze the effect of the external input frequency on the role of gap junctions, uncorrelated inputs with different frequencies were injected into each FSI. For any inputs with different frequencies, the number of spikes reduces rapidly while the synchronization increases slowly with the increase of the gap junction conductance (Figures 3(a) and 3(c) blue). The composition of spikes in FSI network has changed significantly because the addition of gap junctions can remove a large number of asynchronous spikes and trigger a small quantity of synchronous spikes. This trend becomes more severe when the external inputs include more high-frequency components (Figure 3(b) blue) because of the low-pass filtering property of gap junctions. Therefore the increment of the network synchronization becomes smaller with the increase of high-frequency components (Figure 3(c) blue).
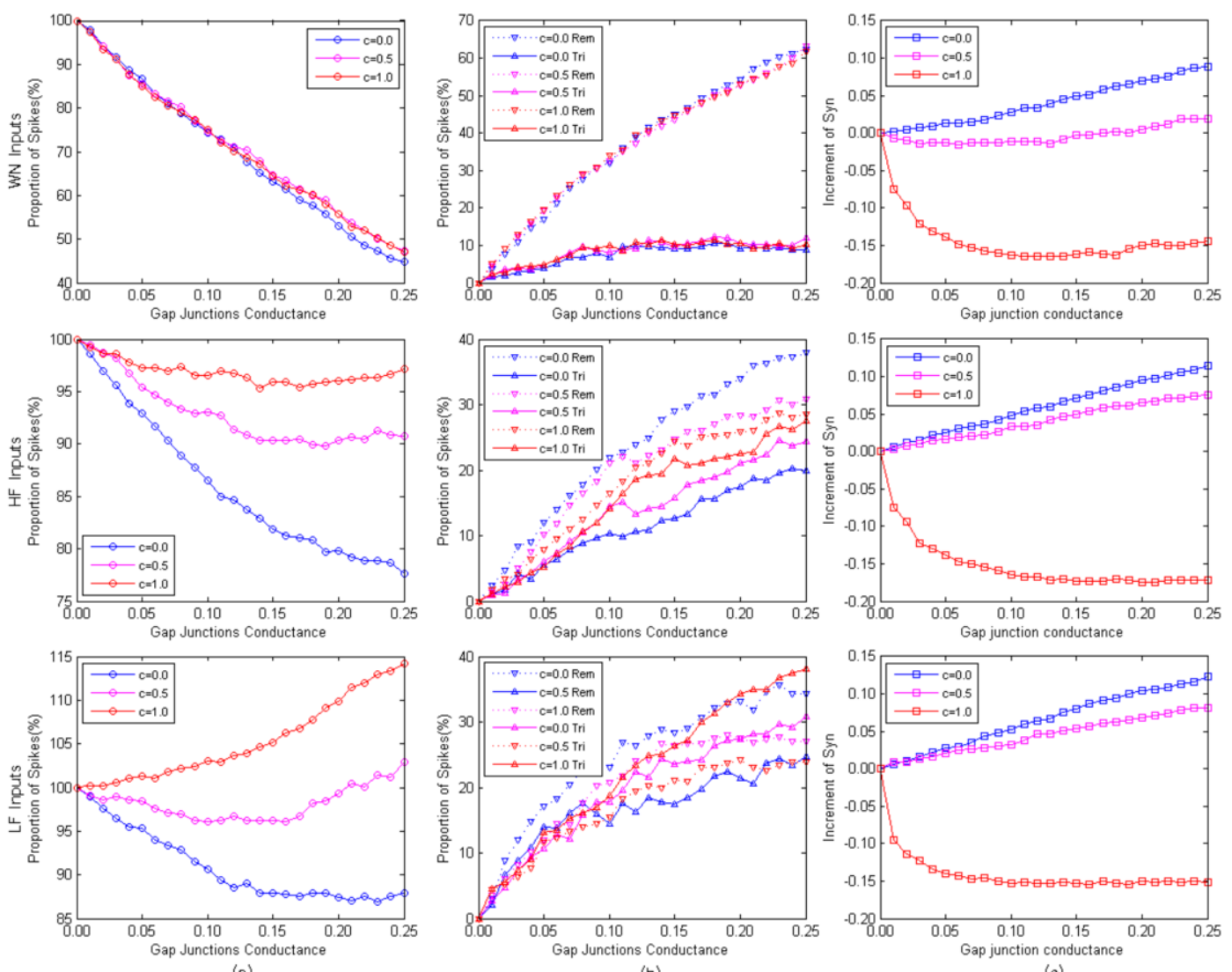

(b)

(c)

Fig. 3. The effect of gap junctions for the different inputs in the simple network. Top: for the white noise inputs; middle: for the high-frequency noise inputs; bottom: for the low-frequency noise inputs. (a) The change of the number of spikes. (b) The change of the number of removed spikes and triggered spikes. (c) The increment of synchronization induced by gap junctions. Rem: the removed spikes; Tri: the triggered spikes. 
In the small-world network, this phenomenon is more obvious due to its complex connectivity. The spike reducing rate grows rapidly but the increment rate of synchronization becomes slow. The influence effectiveness of gap junctions has notable difference between different frequency inputs. For external inputs with more high frequency components, the number of spikes reduces quickly to zero when the coupling strength of gap junctions becomes large enough and the degree of the increment of the network synchronization is slight (Figure 4 blue). Thus, many FSIs are completely or nearly silent and the network remains in a globally-asynchronous state [24].

\subsection{Correlation of external inputs improves the firing activities and synchronization of the network}

The external inputs with different correlation coefficients $(c=0.0, c=0.5, c=1.0)$ were injected into coupled FSIs in the simple network to study the effect of gap junctions. For the external inputs with different frequencies, different correlations exert various effects on the role of gap junctions on firing activities. For the Gaussian white noise inputs, the variation of input correlation almost does not change the effect of gap junctions on the firing activities. With the increase of the conductance of gap junctions, the number of reduced spikes is similar, even the numbers of removed spikes and triggered spikes are almost equal (Figures 3(a) and 3(b), Top). For the high-frequency inputs, the number of reduced spikes induced by gap junctions is related to the correlation of the inputs. With the increasing input correlation, the number of removed spikes decreases while that of triggered spikes increases, thus the spike reducing rate decreases obviously (Figures 3(a) and 3(b), Middle). For the low-frequency inputs, the effect of gap junction changes from reducing spikes to increasing spikes when the correlation between inputs is high enough. Although there are still a number of removed spikes, but the number of triggered spikes becomes much more than that of removed spikes (Figures 3(a) and 3(b), Bottom).
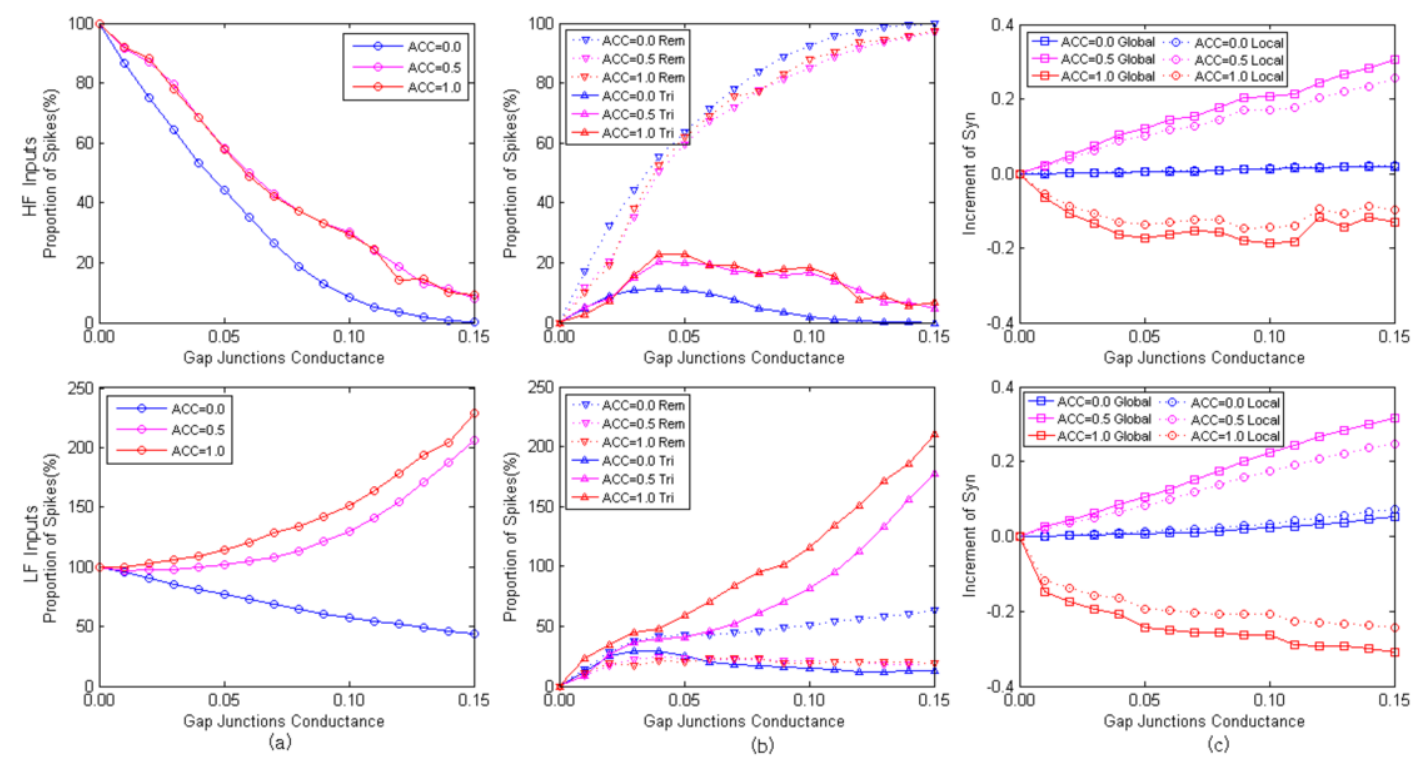

Fig. 4. The effect of gap junctions for the different inputs in NW small-world network. Top: for the high-frequency noise inputs; bottom: for the low-frequency noise inputs. (a) The change of the number of spikes. (b) The change of the number of removed spikes and triggered spikes. (c) The increment of synchronization induced by gap junctions. Rem: the removed spikes; Tri: the triggered spikes. 
For any frequency inputs, the effect of different input correlation on the network synchronization is similar. If the input correlation is less than a certain value, the addition of gap junctions can increase the network synchronization. Although the highly correlated inputs bring the network high synchronization, the increment of synchronization caused by gap junctions is inversely proportional to the input correlation (Figure 3(c)). That is, the rate of synchronization increase is smaller for the higher correlation inputs. But the neuronal network synchronization induced by gap junctions is moderate $[3,6]$, i.e., the maximal synchronization of network is less than the limit value which is determined by the network symmetry rather than the characteristics of the input signals (Figure 3(c)). In general, the factors affecting the symmetry of the network include the connectivity of the network, the conductance of gap junctions and the axon transmission delay. If the input correlation exceeds the limit value, the role of gap junctions will be to decrease rather than increase the network synchronization towards this value. This result differs from that of the previous studies. In fact, the correlation among external inputs has been rarely considered in most of the physiological and model studies and the network synchronization can hardly reach the limit value.

Because of the complex coupling interconnections of the network, the degree of the change in the firing activities and the increment of synchronization are more significant in the small-world network. For the high-frequency inputs, the addition of gap junctions can reduce the number of spikes while the change of $A C C$ has little effect on firing activities when $A C C$ is larger than zero. For the low frequency inputs, the addition of gap junctions can increase the number of spikes when $A C C$ is large enough and the increase of $A C C$ can aggravate the influence on firing activities (Figures 4(a) and 4(b)).

In the NW small-world network, the high and low frequency colored noise with different average correlation coefficients ( $A C C S$ ) was injected into each neuron respectively. Many correlation coefficients between two neuronal inputs will be negative if $A C C$ is zero while all correlation coefficients will be positive in other cases. The increase of $A C C$ can not only make the network highly synchronous but also improve the increment of synchronization obviously when the network synchronization is less than the limit value. However, the relations of local Syn and global Syn are different for different $A C C s$. When the inputs are uncorrelated or less correlated, the local Syn is larger than global Syn, otherwise, the local Syn is smaller than global Syn (Figure 4(c)).

\subsection{The properties of external inputs can affect the firing pattern of the network}

The external inputs with different properties correspond to the stimuli from cortex and thalamus into the FSI network under different conditions in vivo. The external inputs with the low frequency and high correlation are similar to the inputs excited by the behavior-related driving signal. The external inputs with high frequency and low correlation indicate the inputs induced by the background noise.

The addition of the gap junctions reduces or even eliminates the asynchronous spikes to depress the external interference. At the same time, many synchronous spikes are triggered by the gap junctions. The FSIs represent discontinuity bursting when behavioral-linked inputs are applied. Therefore, in the FSI network, the adjustment of spike timing through gap junctions is behavior-related, and the variability of the inter-spike interval (ISI) decreases markedly (Figure 5) [9,10]. The network of striatal FSIs can act as a detector for synchronous input from cortex and thalamus [1]. 



Fig. 5. The change of spiking timing and ISI for the external inputs with the low frequency and high correlation. Left: the firing patterns with different conductance of gap junctions; Right: the corresponding of ISI.

\section{Conclusion}

The gap junctions act as a low-frequency filter [25] which preferentially transmit slow signals better than fast fluctuation which can remove, delay or even add spikes by shunting current [3]. Therefore, the external inputs with different frequencies and correlation can affect the role of gap junctions. For uncorrelated inputs, the major role of the gap junctions is to suppress the firing activity of FSIs in the network by removing asynchronous spikes through the shunting currents. This characteristic of the gap junctions can effectively suppress the high frequency disturbance and help to select relevant information from the cortex and thalamus [1].

The degree of the correlation among the inputs affects the role of the gap junctions in regulating the firing activities of the neuronal network. For the Gaussian white noise inputs, the change of the input correlation has no effect on the role of gap junctions which can be due to the nonlinear properties and transmission delay of gap junctions. For the high-frequency noise inputs, the increase of input correlation significantly lessens the reduction of spikes. That means highly correlated inputs can effectively reduce the suppression of firing activities [3]. For the low-frequency noise inputs, high correlation of inputs largely increases the number of spikes because of a lot of triggered spikes. This phenomenon is consistent with the enhancement of the network firing activities when the behavior-link stimuli is received [10]. The asymmetry of the network makes its synchronization remain below the limit value. If the network synchronization is less than the limit value, the gap junctions can bring a smaller degree of synchronization increment due to the higher correlation. When the synchronization is high than the limit value, the gap junctions can cut down the synchrony.

Compared with the simple network, the role of the gap junctions in the NW small-world network is significantly enhanced due to the complex connectivity. The relation of global and local synchronization is related to the input correlation. If the input correlation is low, the local synchronization is generally higher than the global one because of the high-clustering property of the 
network; otherwise, the global synchronization may be higher than the local one when the correlation of inputs is high.

In conclusion, for the uncorrelated high-frequency inputs, the gap junctions can slightly promote the network synchrony and suppress the firing activities. For the correlated low-frequency inputs, the gap junctions can improve the synchrony and enhance the firing activities obviously. Thus, the effect of the gap junctions on FSI network is to effectively filter out the background noise and detect the synchronous input from cortex and thalamus [1].

\section{Acknowledgement}

This work was supported by the National Natural Science Foundation of China (No. 61271088).

\section{References}

[1] E. Fino and L. Venance, Spike-timing dependent plasticity in striatal interneurons, Neuropharmacology 60 (2011), $780-788$.

[2] T. Koos and J.M. Tepper, Inhibitory control of neostriatal projection neurons by GABAergic interneurons, Nature Neuroscience 2 (1999), 467-472.

[3] J. Hjorth, K.T. Blackwell, and J.H. Kotaleski, Gap Junctions between striatal fast-spiking interneurons regulate spiking activity and synchronization as a function of cortical activity, Journal of Neuroscience 29 (2009), 5276-5286.

[4] G.J. Gage, et al., Selective activation of striatal fast-spiking interneurons during choice execution, Neuron. 67 (2010), 466-479.

[5] J.M. Tepper, T. Koos and C.J. Wilson, GABAergic microcircuits in the neostriatum, Trends in Neurosciences 27 (2004), $662-669$.

[6] J.R. Gibson, M. Beierlein and B.W. Connors, Functional properties of electrical synapses between inhibitory interneurons of neocortical layer 4, Journal of Neurophysiology 93 (2005), 467-480.

[7] A.H. Gittis et al., Distinct roles of GABAergic interneurons in the regulation of striatal output pathways, Journal of Neuroscience 30 (2010), 2223-2234.

[8] M. Galarreta and S. Hestrin, A network of fast-spiking cells in the neocortex connected by electrical synapses, Nature 402 (1999), 72-75.

[9] J.D. Berke, Uncoordinated firing rate changes of striatal fast-spiking interneurons during behavioral task performance, Journal of Neuroscience 28 (2008), 10075-10080.

[10] J.D. Berke, Functional properties of striatal fast-spiking interneurons, Frontiers in systems neuroscience 5 (2011), 45.

[11] J.B. Ding et al., Thalamic gating of corticostriatal signaling by cholinergic interneurons, Neuron. 67 (2010), 294-307.

[12] S. Ramanathan et al., Synaptic convergence of motor and somatosensory cortical afferents onto GABAergic Interneurons in the rat striatum, Journal of Neuroscience 22 (2002), 8158-8169.

[13] Q.Y. Wang, Q.S. Lu and G.R. Chen, Ordered bursting synchronization and complex wave propagation in a ring neuronal network, Physica a-Statistical Mechanics and Its Applications 374 (2007), 869-878.

[14] H. Kitajima and J. Kurths, Synchronized firing of fitzhugh-nagumo neurons by noise, Chaos. 15 (2005), 1-5.

[15] H. Yu et al., Effects of hybrid synapses on the vibrational resonance in small-world neuronal networks, Chaos. 22 (2012), 033105-1-033105-8.

[16] R.F. Fox et al., Fast, accurate algorithm for numerical-simulation of exponentially correlated colored noise, Physical Review A 38 (1988), 5938-5940.

[17] E.M. Izhikevich, Hybrid spiking models, Philosophical Transactions of the Royal Society a-Mathematical Physical and Engineering Sciences 368 (2010), 5061-5070.

[18] E.M. Izhikevich, Simple model of spiking neurons, Ieee Transactions on Neural Networks 14 (2003), 1569-1572.

[19] M.D. Humphries, R. Wood and K. Gurney, Dopamine-modulated dynamic cell assemblies generated by the GABAergic striatal microcircuit, Neural Networks 22 (2009), 1174-1188.

[20] M.E.J. Newman and D.J. Watts, Renormalization group analysis of the small-world network model, Phys. Lett. A 263 (1999), 341-346. 
[21] F. Han et al., Firing synchronization of learning neuronal networks with small-world connectivity, International Journal of Non-Linear Mechanics 47 (2012), 1161-1166.

[22] D. Guo, Q. Wang and M. Perc, Complex synchronous behavior in interneuronal networks with delayed inhibitory and fast electrical synapses, Physical Review E 85 (2012), 061905-1-061905-8.

[23] K. Kube et al., Spike-timing-dependent plasticity in small-world networks, Neurocomputing 71 (2008), 1694-1704.

[24] M.D. Humphries, R. Wood and K. Gurney, Reconstructing the three-dimensional GABAergic microcircuit of the striatum, Plos Computational Biology 6 (2010), 1-11.

[25] M. Galarreta and S. Hestrin, Electrical synapses between GABA-releasing interneurons, Nature Reviews Neuroscience 2 (2001), 425-433. 